\title{
Distances and Similarity Measures in Heuristic Possibilistic Clustering the Intuitionistic Fuzzy Data: A Comparative Study
}

\author{
Dmitri A. Viattchenin ${ }^{1, *}$, Stanislav Shiray ${ }^{2}$ \\ ${ }^{1}$ Laboratory of System Identification, United Institute of Informatics Problems of the National Academy of Sciences of Belarus, Minsk, \\ Belarus \\ ${ }^{2}$ Department of Software Information Technology, Belarusian State University of Informatics and Radio-Electronics, Minsk, Belarus
}

\section{Email address:}

viattchenin@mail.ru (D. A. Viattchenin), ashaman410@gmail.com (S. Shiray)

*Corresponding author

\section{To cite this article:}

Dmitri A. Viattchenin, Stanislav Shiray. Distances and Similarity Measures in Heuristic Possibilistic Clustering the Intuitionistic Fuzzy Data: A Comparative Study. International Journal of Sustainability Management and Information Technologies. Vol. 3, No. 6, 2017 , pp. 57-62. doi: 10.11648/j.ijsmit.20170306.11

Received: February 28, 2017; Accepted: March 22, 2017; Published: December 14, 2017

\begin{abstract}
The note deals with the problem of heuristic possibilistic clustering the intuitionistic fuzzy data. Different distances between intuitionistic fuzzy sets are considered in the paper. Similarity measures for intuitionistic fuzzy sets for constructing intuitionistic fuzzy tolerance relations are also considered. A numerical example of application of these distances and similarity measures for clustering the intuitionistic fuzzy data is presented. Some preliminary conclusions are formulated.
\end{abstract}

Keywords: Clustering, Intuitionistic Fuzzy Data, Distance, Similarity Measure, Allotment Among Intuitionistic Fuzzy Clusters

\section{Introduction}

Cluster analysis is a group of approaches for classifying objects according to their likeness by means of unsupervised training. It makes the objects, which have greater likeness, as a class, or cluster, and occupies the partial area of feature space. The cluster prototype of each partial area is respectively acting as a representative of the corresponding type. Fuzzy sets theory, which was proposed by Zadeh [1], makes it possible to model partial belongingness to a cluster, which is described by a membership function. Fuzzy clustering methods have been applied effectively in image processing, data analysis, symbol recognition and modeling. Moreover, fuzzy set theory is a basis for possibility theory [2]. Thus, a possibilistic approach to clustering was proposed by Krishnapuram and Keller in [3] and developed by other researchers. A concept of possibilistic partition is a basis of possibilistic clustering methods and the membership values can be interpreted as the values of typicality degree. Fuzzy and possibilistic clustering methods are considered at length, for instance, in [4-6].
The most common and widespread approach to fuzzy clustering is the optimization approach. Moreover, major possibilistic clustering methods are also objective functionbased clustering algorithms. However, heuristic algorithms of fuzzy clustering are simple and very effective in many cases, because heuristic algorithms display high level of essential clarity and low level of complexity. Some heuristic clustering procedures are based on the definition of a cluster concept and the purpose of these algorithms is cluster detection conform to a given definition. Such algorithms are called algorithms of direct classification or direct clustering algorithms. Thus, a heuristic approach to possibilistic clustering in which the sought clustering structure of the set of objects is based directly on the formal definition of fuzzy $\alpha$-cluster and possibilistic memberships are determined directly from the values of pairwise similarity of objects was proposed in [7] and developed in other publications. The essence of the heuristic approach to possibilistic clustering is that the sought clustering structure of the set of observations is formed based directly on the formal definition of fuzzy cluster and possibilistic memberships are determined also directly from the values of the pairwise similarity of 
observations. A concept of the allotment among fuzzy clusters is basic concept of the approach and the allotment among fuzzy clusters is a special case of the possibilistic partition.

Direct heuristic algorithms of possibilistic clustering can be divided into two types: relational versus prototype-based. A fuzzy tolerance relation $T$ matrix is a matrix of the initial data for the direct heuristic relational algorithms of possibilistic clustering and a matrix of attributes is a matrix of the initial data for the prototype-based algorithms. In particular, the group of direct relational heuristic algorithms of possibilistic clustering includes

a) the D-AFC(c)-algorithm which is based on the construction of an allotment $R_{c}^{*}(X)$ among an a priori given number $c$ of partially separate fuzzy $\alpha$-clusters;

b) the D-PAFC-algorithm which is based on the construction of an principal allotment $R_{P}^{*}(X)$ among an unknown minimal number of at least $c$ fully separate fuzzy $\alpha$-clusters;

c) the D-AFC-PS(c)-algorithm which is based on the construction of an allotment $R_{c}^{*}(X)$ among an a priori given number $c$ of partially separate fuzzy $\alpha$-clusters in the presence of labeled objects.

On the other hand, the family of direct prototype-based heuristic algorithms of possibilistic clustering consists of

a) the D-AFC-TC-algorithm which is based on the construction of an allotment $R_{c}^{*}(X)$ among an a priori unknown number $c$ of fully separate fuzzy $\alpha$-clusters;

b) the D-PAFC-TC-algorithm which is based on the construction of a principal allotment $R_{P}^{*}(X)$ among an a priori unknown minimal number of at least $c$ fully separate fuzzy $\alpha$-clusters;

c) the D-AFC-TC $(\alpha)$-algorithm which is based on the construction of an allotment $R_{c}^{*}(X)$ among an a priori unknown number $c$ of fully separate fuzzy $\alpha$-clusters with respect to the minimal value $\alpha$ of the tolerance threshold.

It should be noted, that these prototype-based heuristic algorithms of possibilistic clustering are based on the transitive closure of the initial fuzzy tolerance.

Since the fundamental Atanassov's paper [8] was published, intuitionistic fuzzy sets theory has been applied to many areas such as learning, decision-making and classification. Techniques for clustering the intuitionistic fuzzy data were proposed by different researchers and these algorithms are summarized in [9]. However, the intuitionistic fuzzy set-based extension of the heuristic approach to possibilistic clustering was also outlined in [7]. Direct heuristic algorithms of possibilistic clustering for processing the intuitionistic fuzzy data can be also divided into two types: relational versus prototype-based. An intuitionistic fuzzy tolerance relation matrix is a matrix of the initial data for the relational algorithms and a matrix of attributes is a matrix of the initial data for the prototype-based algorithms. In particular, the group of direct relational heuristic algorithms of possibilistic clustering the intuitionistic fuzzy data contains

a) the D-PAIFC-algorithm which is based on the construction of an principal allotment $I R_{P}^{*}(X)$ among a priori unknown minimal number at least $c$ fully separate intuitionistic fuzzy $(\alpha, \beta)$-clusters [7];

b) the D-AIFC(c)-algorithm which is based on the construction of an allotment $I_{c}^{*}(X)$ among a priori given number $c$ of partially separate intuitionistic fuzzy $(\alpha, \beta)$-clusters [10].

The group of direct prototype-based heuristic algorithms of possibilistic clustering the intuitionistic fuzzy data is formed by

a) the D-PAIFC-TC-algorithm which is based on the construction of a principal allotment $I R_{P}^{*}(X)$ among a priori unknown minimal number at least $c$ fully separate intuitionistic fuzzy $(\alpha, \beta)$-clusters [11];

b) the D-AIFC-TC-algorithm which is based on the construction of an allotment $I_{c}^{*}(X)$ among a priori unknown number $c$ of fully separate intuitionistic fuzzy $(\alpha, \beta)$-clusters [12].

It should be noted, that these prototype-based heuristic algorithms of possibilistic clustering are based on the transitive closure of the initial intuitionistic fuzzy tolerance. The corresponding procedure is proposed in [13].

The main purpose of the presented paper is a comparative analysis of application of different distances and similarity measures between intuitionistic fuzzy sets for clustering the intuitionistic fuzzy data by using heuristic algorithms of possibilistic clustering. In particular, the D-PAIFC-algorithm and the D-PAFC-algorithm were selected for the comparison. Thus, the contents of this paper are the following: in the second section some definitions of the intuitionistic fuzzy set theory are described, in the third section distances between intuitionistic fuzzy sets are presented, in the fourth section similarity measures for constructing intuitionistic fuzzy tolerance relation are described, in the fifth section results of numerical experiments are presented, in sixth section some preliminary conclusions are formulated and perspectives of future investigations are outlined.

\section{Basic Definitions of the Intuitionistic Fuzzy Set Theory}

The intuitionistic fuzzy sets were developed by Atanassov also in [14], [15] and other researchers as an extension of the ordinary fuzzy sets. Let us remind some basic definitions of the intuitionistic fuzzy sets theory which will be used in further considerations. All concepts will be presented for a finite universe $X=\left\{x_{1}, \ldots, x_{n}\right\}$.

An intuitionistic fuzzy set $I A$ in $X$ is given by ordered triple $I A=\left\{\left\langle x_{i}, \mu_{I A}\left(x_{i}\right), v_{I A}\left(x_{i}\right)\right\rangle \mid x_{i} \in X\right\}$, where $\mu_{I A}, v_{I A}$ : $X \rightarrow[0,1]$ should satisfy a condition

$$
0 \leq \mu_{I A}\left(x_{i}\right)+v_{I A}\left(x_{i}\right) \leq 1
$$


for all $x_{i} \in X$. The values $\mu_{I A}\left(x_{i}\right)$ and $v_{I A}\left(x_{i}\right)$ denote the degree of membership and the degree of non-membership of element $x_{i} \in X$ to $I A$, respectively. For each intuitionistic fuzzy set $I A$ in $X$ an intuitionistic fuzzy index of an element $x_{i} \in X$ in $I A$ can be defined as follows

$$
\rho_{I A}\left(x_{i}\right)=1-\left(\mu_{I A}\left(x_{i}\right)+v_{I A}\left(x_{i}\right)\right) \text {. }
$$

The intuitionistic fuzzy index $\rho_{I A}\left(x_{i}\right)$ can be considered as a hesitancy degree of $x_{i}$ to $I A$. It is seen that $0 \leq \rho_{I A}\left(x_{i}\right) \leq 1$ for all $x_{i} \in X$. Obviously, when $v_{I A}\left(x_{i}\right)=1-\mu_{I A}\left(x_{i}\right)$ for every $x_{i} \in X$, the intuitionistic fuzzy set $I A$ is an ordinary fuzzy set in $X$. For each ordinary fuzzy set $A$ in $X$, we have $\rho_{A}\left(x_{i}\right)=0$, for all $x_{i} \in X$.

The binary intuitionistic fuzzy relation $I R$ on $X$ is an intuitionistic fuzzy subset $I R$ of $X \times X$, which is given by the expression

$$
I R=\left\{\left\langle\left(x_{i}, x_{j}\right), \mu_{I R}\left(x_{i}, x_{j}\right), v_{I R}\left(x_{i}, x_{j}\right)\right\rangle \mid x_{i}, x_{j} \in X\right\},
$$

where $\mu_{I R}: X \times X \rightarrow[0,1]$ and $v_{I R}: X \times X \rightarrow[0,1]$ satisfy the condition $0 \leq \mu_{I R}\left(x_{i}, x_{j}\right)+v_{I R}\left(x_{i}, x_{j}\right) \leq 1$ for every $\left(x_{i}, x_{j}\right) \in X \times X$.

An intuitionistic fuzzy relation $\operatorname{IR} \in \operatorname{IFR}(X)$ is reflexive if for every $x_{i} \in X, \mu_{I R}\left(x_{i}, x_{i}\right)=1$ and $v_{I R}\left(x_{i}, x_{i}\right)=0$. An intuitionistic fuzzy relation $\operatorname{IR} \in \operatorname{IFR}(X)$ is called symmetric if for all $\left(x_{i}, x_{j}\right) \in X \times X \quad, \quad$ a condition $\mu_{I R}\left(x_{i}, x_{j}\right)=\mu_{I R}\left(x_{j}, x_{i}\right) \quad$ and a condition $v_{I R}\left(x_{i}, x_{j}\right)=v_{I R}\left(x_{j}, x_{i}\right)$ are met. An intuitionistic fuzzy relation $I T$ in $X$ is called an intuitionistic fuzzy tolerance if it is reflexive and symmetric.

\section{Some Distances Between Intuitionistic Fuzzy Sets}

Let us remind some distances between intuitionistic fuzzy sets which were proposed by Szmidt and Kasprzyk in different publications and summarized in [16]. In particular, for two intuitionistic fuzzy sets $I A$ and $I B$ in $X$ the following distances were proposed:

- the normalized Hamming distance:

$$
l_{(I F S)}(A, B)=\frac{1}{2 n} \sum_{i=1}^{n}\left(\left|\mu_{A}\left(x_{i}\right)-\mu_{B}\left(x_{i}\right)\right|+\left|v_{A}\left(x_{i}\right)-v_{B}\left(x_{i}\right)\right|+\left|\rho_{A}\left(x_{i}\right)-\rho_{B}\left(x_{i}\right)\right|\right)
$$

- the normalized Hausdorff distance:

$$
h_{(I I F)}(A, B)=\frac{1}{2 n} \sum_{i=1}^{n} \max \left(\left|\mu_{A}\left(x_{i}\right)-\mu_{B}\left(x_{i}\right)\right|,\left|v_{A}\left(x_{i}\right)-v_{B}\left(x_{i}\right)\right|,\left|\rho_{A}\left(x_{i}\right)-\rho_{B}\left(x_{i}\right)\right|\right)
$$

- the normalized Euclidean distance:

$$
e_{(I F S)}(A, B)=\sqrt{\frac{1}{2 n} \sum_{i=1}^{n}\left(\left(\mu_{A}\left(x_{i}\right)-\mu_{B}\left(x_{i}\right)\right)^{2}+\left(v_{A}\left(x_{i}\right)-v_{B}\left(x_{i}\right)\right)^{2}+\left(\rho_{A}\left(x_{i}\right)-\rho_{B}\left(x_{i}\right)\right)^{2}\right)}
$$

These distances satisfy the conditions of the metric [16]. A unique value of dissimilarity between intuitionistic fuzzy sets $I A$ and $I B$ in $X$ is the result of application formulas (4) (6) to the intuitionistic fuzzy sets. So, a value of similarity between intuitionistic fuzzy sets $I A$ and $I B$ can be calculated from the complement operation [17]

$$
S_{(I F S)}(A, B)=1-d_{(I F S)}(A, B),
$$

where $d_{(I F S)}(A, B)$ is a general notation for the distances (4) $-(6)$.

\section{Similarity Measures for Constructing Intuitionistic Fuzzy Tolerances}

The method for constructing the intuitionistic fuzzy tolerance relation from a family of intuitionistic fuzzy sets was proposed by Wang, Xu, Liu and Tang in [18]. The corresponding similarity measure is based on the normalized Hamming distance and the similarity measure can be expressed by a formula

$$
S_{l(I F S)}(I A, I B)=\left\{\begin{array}{ll}
(1,0), & I A=I B \\
\left(1-\frac{1}{n} \sum_{i=1}^{n}\left|v_{I A}\left(x_{i}\right)-v_{I B}\left(x_{i}\right)\right|-\frac{1}{n} \sum_{i=1}^{n}\left|\rho_{I A}\left(x_{i}\right)-\rho_{I B}\left(x_{i}\right)\right|,\right. &
\end{array}\right), I A \neq I B,
$$

for all $i=1, \ldots, n$. That is why the closeness degree $s_{l(I F S)}(I A, I B)=\left(\mu_{l(I F S)}(I A, I B), v_{l(I F S)}(I A, I B)\right)$ of intuitionistic fuzzy sets $I A$ and $I B$ can be constructed according to the formula (8). Obviously, if all the differences of values of the non-membership degree and the differences of values of the intuitionistic fuzzy index of two objects $I A$ and $I B$ with respect to attributes $x_{i}, i=1, \ldots, n$ get smaller, then the two objects are more similar to each other for all $i=1, \ldots, n$.

The corresponding intuitionistic fuzzy relation possesses the symmetry property and the reflexivity property. Moreover, the condition $0 \leq \mu_{l(I F S)}(I A, I B)+v_{l(I F S)}(I A, I B) \leq 1$ is met for any intuitionistic fuzzy sets $I A$ and $I B$. These facts were proved in [18].

On the other hand, a similarity measure based on the normalized Hausdorff distance was proposed in [19]. The similarity measure can be written as follows:

$$
s_{h(I F S)}(I A, I B)=\left(\begin{array}{l}
1-\frac{1}{n} \sum_{i=1}^{n} \max \left\{\left|v_{I A}\left(x_{i}\right)-v_{I B}\left(x_{i}\right)\right|,\left|\rho_{I A}\left(x_{i}\right)-\rho_{I B}\left(x_{i}\right)\right|\right\}, \\
\frac{1}{n} \sum_{i=1}^{n} \max \left|v_{I A}\left(x_{i}\right)-v_{I B}\left(x_{i}\right)\right|
\end{array}\right) .
$$

A similarity measure based on the normalized Euclidean distance was proposed in [20] and the corresponding similarity measure is defined by a formula

$$
S_{e(I F S)}(I A, I B)=\left(\begin{array}{l}
1-\frac{1}{2 n} \sum_{i=1}^{n} \sqrt{\left(v_{I A}\left(x_{i}\right)-v_{I B}\left(x_{i}\right)\right)^{2}+\left(\rho_{I A}\left(x_{i}\right)-\rho_{I B}\left(x_{i}\right)\right)^{2}}, \\
\frac{1}{2 n} \sum_{i=1}^{n} \sqrt{\left(v_{I A}\left(x_{i}\right)-v_{I B}\left(x_{i}\right)\right)^{2}}
\end{array}\right) .
$$


The closeness degrees $S_{(I F S)}(I A, I B)$ of intuitionistic fuzzy sets $I A$ and $I B$ satisfy the condition $0 \leq \mu_{(I F S)}(I A, I B)+v_{(I F S)}(I A, I B) \leq 1 \quad, \quad$ where $s_{(I F S)}(I A, I B)=\left(\mu_{(I F S)}(I A, I B), v_{(I F S)}(I A, I B)\right)$ is a general notation for the similarity measures (9), (10). The corresponding intuitionistic fuzzy relations satisfied to the symmetry property and the reflexivity property. That is why the intuitionistic fuzzy relations are intuitionistic fuzzy tolerances.

\section{Numerical Experiments}

Let us consider application of the considered distances and similarity measures between intuitionistic fuzzy sets to solving the classification problem. For the purpose, Wang's cars data set [18] were used. The data set contains the information of ten new cars $x_{i}, i=1, \ldots, 10$ to be classified into several kinds. Each car has six evaluation attributes which represent the oil consumption, coefficient of friction, price, comfortable degree, design and safety coefficient evaluated for five cars. Denote oil consumption by $x^{1}$, coefficient of friction by $x^{2}$, price by $x^{3}$, comfortable degree by $x^{4}$, design by $x^{5}$ and safety coefficient by $x^{6}$. The characteristics of cars under the six factors $x^{t_{1}}$, $t_{1}=1, \ldots, 6$ are represented by the intuitionistic fuzzy sets, as shown in Table 1. Thus, each car can be interpreted as an intuitionistic fuzzy set $x_{i}, i=1, \ldots, 10$ on the universe of attributes $x^{t_{1}}, t_{1}=1, \ldots, 6$.

Table 1. The initial intuitionistic fuzzy data.

\begin{tabular}{lllllll}
\hline \multirow{2}{*}{ Cars } & \multicolumn{2}{l}{ Factors } & \multicolumn{1}{l}{$l$} \\
\cline { 2 - 7 } & $\boldsymbol{x}^{\mathbf{1}}$ & $\boldsymbol{x}^{\mathbf{2}}$ & $\boldsymbol{x}^{3}$ & $\boldsymbol{x}^{4}$ & $\boldsymbol{x}^{5}$ & $\boldsymbol{x}^{6}$ \\
\hline$x_{1}$ & $(0.8,0.1)$ & $(0.4,0.1)$ & $(0.6,0.1)$ & $(0.7,0.3)$ & $(0.6,0.2)$ & $(0.5,0.0)$ \\
$x_{2}$ & $(0.0,0.3)$ & $(0.1,0.3)$ & $(0.0,0.6)$ & $(0.0,0.5)$ & $(0.5,0.3)$ & $(0.4,0.2)$ \\
$x_{3}$ & $(0.2,0.0)$ & $(0.9,0.1)$ & $(0.0,0.7)$ & $(0.0,0.1)$ & $(0.3,0.2)$ & $(0.8,0.2)$ \\
$x_{4}$ & $(0.0,0.5)$ & $(0.3,0.0)$ & $(0.7,0.1)$ & $(0.6,0.1)$ & $(0.0,0.7)$ & $(0.7,0.2)$ \\
$x_{5}$ & $(0.4,0.6)$ & $(0.2,0.4)$ & $(0.9,0.1)$ & $(0.6,0.1)$ & $(0.7,0.2)$ & $(0.7,0.3)$ \\
$x_{6}$ & $(0.0,0.2)$ & $(0.0,0.0)$ & $(0.5,0.4)$ & $(0.5,0.4)$ & $(0.3,0.6)$ & $(0.0,0.0)$ \\
$x_{7}$ & $(0.8,0.1)$ & $(0.2,0.1)$ & $(0.1,0.0)$ & $(0.7,0.0)$ & $(0.6,0.4)$ & $(0.0,0.6)$ \\
$x_{8}$ & $(0.1,0.7)$ & $(0.0,0.5)$ & $(0.8,0.1)$ & $(0.7,0.1)$ & $(0.7,0.1)$ & $(0.0,0.0)$ \\
$x_{9}$ & $(0.0,0.1)$ & $(0.5,0.1)$ & $(0.3,0.1)$ & $(0.7,0.3)$ & $(0.1,0.3)$ & $(0.7,0.2)$ \\
$x_{10}$ & $(0.3,0.2)$ & $(0.7,0.1)$ & $(0.2,0.2)$ & $(0.2,0.0)$ & $(0.1,0.9)$ & $(0.9,0.1)$ \\
\hline
\end{tabular}

Matrices of ordinary fuzzy tolerance relations were constructed according to formulas (4) - (6) and (7). So, the D-PAFC-algorithm can be applied to each fuzzy tolerance. Let us consider the results of numerical experiments.

By executing the D-PAFC-algorithm for the fuzzy tolerance relation obtained by using the normalized Hamming distance (4) and the normalized Hausdorff distance (5), we obtain the principal allotment $R_{P}^{\alpha}(X)$ among two fuzzy clusters. Membership values of two classes, obtained from the D-PAFC-algorithm, are presented in figures 1 and 2, where membership values of the first class are represented by $\square$ and membership values of the second class are represented by $\circ$.

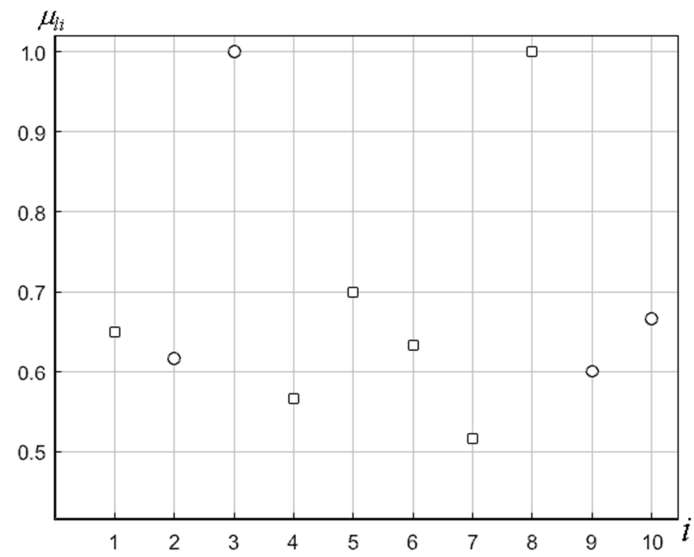

Figure 1. Membership functions of two fuzzy clusters obtained from the DPAFC-algorithm by using the normalized Hamming distance (4).

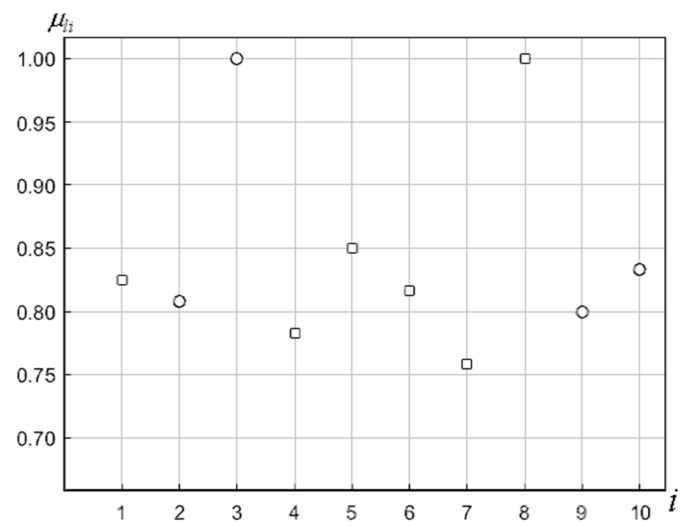

Figure 2. Membership functions of two fuzzy clusters obtained from the DPAFC-algorithm by using the normalized Hausdorff distance (5).

Thus, we obtain the following: the first class is composed by six elements and the second class consists of four elements in both cases. The eighth object is the typical point of the first intuitionistic fuzzy cluster and the third object is the typical point of the second intuitionistic fuzzy cluster. The principal allotment was obtained for the tolerance threshold $\alpha=0.5167$ in the case of the distance (4) and $\alpha=0.7583$ in the case of the distance (5).

By executing the D-PAFC-algorithm for the fuzzy tolerance relation obtained by using the normalized Euclidean distance (6) we obtain the principal allotment $R_{P}^{\alpha}(X)$ among four fuzzy clusters for the tolerance threshold $\alpha=0.6063$. Membership values of four classes, obtained from the D-PAIFC-algorithm, are presented in figure 3, where membership values of the first class are represented by $\circ$, membership values of the second class are represented by $\square$, membership values of the third class are represented by $\diamond$, membership values of the fourth class are represented by $\Delta$. 


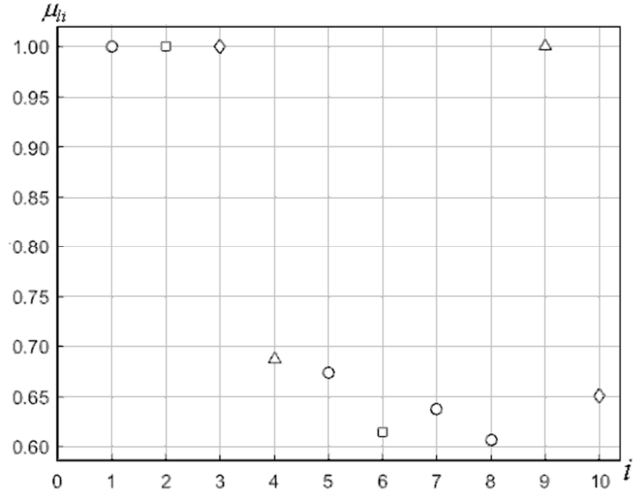

Figure 3. Membership functions of four fuzzy clusters obtained from the DPAFC-algorithm by using the normalized Euclidean distance (6).

On the other hand, intuitionistic fuzzy tolerances were constructed according to formulas (8) - (10) and the DPAIFC-algorithm was applied to each intuitionistic fuzzy tolerance relation. After application of the D-PAIFCalgorithm to matrices of intuitionistic fuzzy tolerance, the principal allotment $I R_{P}^{\alpha, \beta}(X)$ among sixth intuitionistic fuzzy clusters, which corresponds to the classification result, was received in each case.

Membership values and non-membership values of six classes, obtained from the D-PAIFC-algorithm, are presented in figures $4-6$, where membership values of the first class are represented by $\triangleleft$, membership values of the second class are represented by $\circ$, membership values of the third class are represented by $\square$, membership values of the fourth class are represented by $\Delta$, membership values of the fifth class are represented by $\nabla$, and membership values of the sixth class are represented by $\diamond$. Non-membership values of the first class are represented by 4 , non-memberships of the second class are represented by $\bullet$, non-memberships of the third class are represented by $\mathbf{m}$, non-memberships of the fourth class are represented by $\boldsymbol{\Lambda}$, and non-memberships of the fifth class are represented by $\boldsymbol{\nabla}$, and non-membership values of the sixth class are represented by $\bullet$.

Membership values and non-membership values of residual elements of intuitionistic fuzzy clusters are not shown in figures $4-6$.

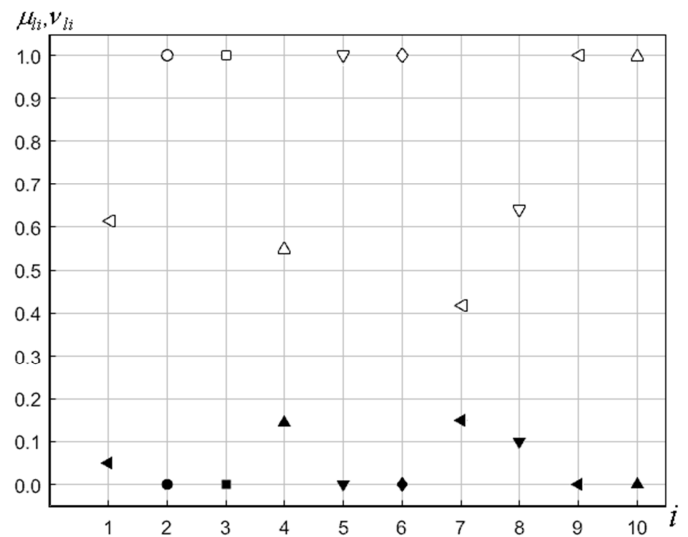

Figure 4. Membership functions and non-membership functions of sixth intuitionistic fuzzy clusters obtained from the D-PAIFC-algorithm by using the similarity measure based on the normalized Hamming distance (8).

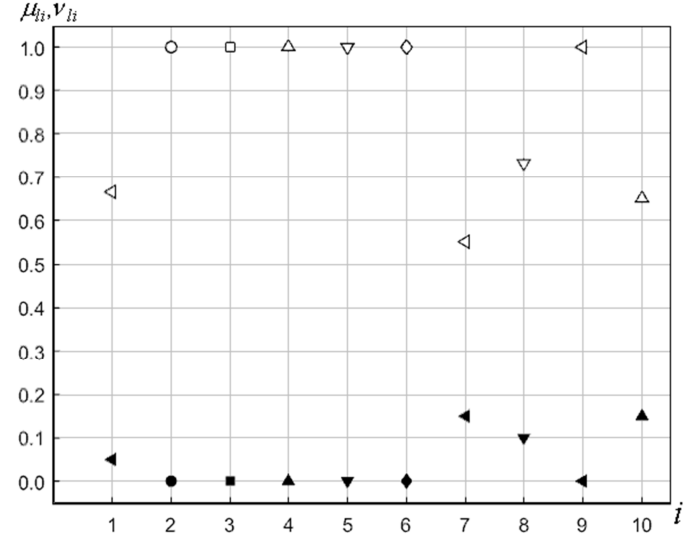

Figure 5. Membership functions and non-membership functions of sixth intuitionistic fuzzy clusters obtained from the D-PAIFC-algorithm by using the similarity measure based on the normalized Hausdorff distance (9).

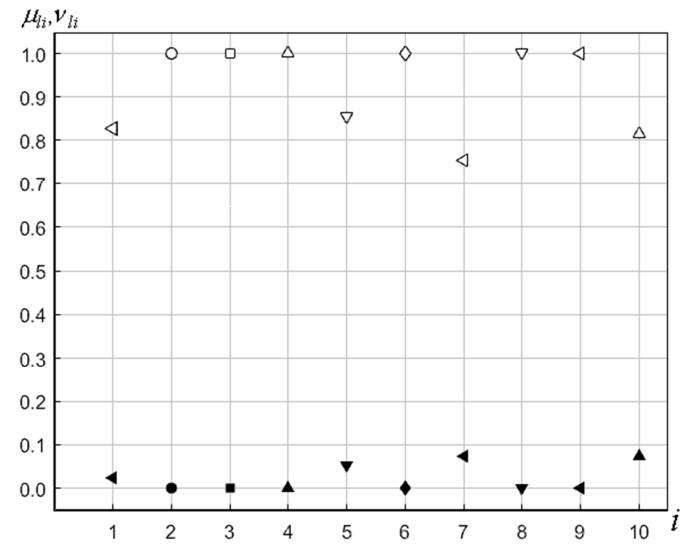

Figure 6. Membership functions and non-membership functions of sixth intuitionistic fuzzy clusters obtained from the D-PAIFC-algorithm by using the similarity measure based on the normalized Euclidean distance (10).

So, we obtain the following: the first class is formed by three elements, the second class is composed of one element, the third class consists of one element, the fourth class contains two elements, the fifth class is composed of two elements, and the sixth class is formed by one element. The second object, the third object, the tenth object, the fifth object, the sixth object, and the ninth object are typical points of corresponding intuitionistic fuzzy clusters in the first case, the second object, the third object, the fourth object, the fifth object, the sixth object and the ninth object are typical points of corresponding intuitionistic fuzzy clusters in the second case, and the second object, the third object, the fourth object, the eighth object, the sixth object, and the ninth object are typical points of intuitionistic fuzzy clusters in the third case.

\section{Concluding Remarks}

The differences between heuristic possibilistic clustering results obtained from the D-PAFC-algorithm by using wellknown distances between intuitionistic fuzzy sets and from the D-PAIFC-algorithm by using similarity measures for constructing intuitionistic fuzzy tolerances are shown in the 
paper. A principal allotment among fuzzy clusters is the result of application of the conventional D-PAFC-algorithm of the heuristic approach to possibilistic clustering to classification the attributive intuitionistic fuzzy data by using distances between intuitionistic fuzzy sets. A principal allotment among intuitionistic fuzzy clusters is the result of application of the D-PAIFC-algorithm to classification the data which can be obtained by using similarity measures. So, non-membership values of objects are also presented in the case. That is why the use of the D-PAIFC-algorithm in combination with the similarity measures is more preferred than use of the D-PAFC-algorithm in combination with distances between intuitionistic fuzzy sets.

Both approaches to clustering the intuitionistic fuzzy data were tested on the Wang's cars data set [18]. The results of applying the D-PAFC-algorithm in combination with the distances between intuitionistic fuzzy sets differ from each other for different distances. On the other hand, the results of applying the D-PAIFC-algorithm in combination with the similarity measures are resemble in all cases. However, the difference of results is not sufficient for preferences for one approach to clustering the intuitionistic fuzzy data before another approach. Therefore, experiments must be performed for other data sets in further studies.

\section{Acknowledgements}

Authors are grateful to Prof. Janusz Kacprzyk, Prof. Eulalia Szmidt, and Dr. Jan W. Owsiński for their interest in our investigations and support. We would like to thank the anonymous referees for their valuable comments and remarks. We express our deep gratitude to the editorial board of the Journal for their wonderful cooperation.

\section{References}

[1] L. A. Zadeh, "Fuzzy sets", Information and Control, 8 (3), pp. 338-353, 1965.

[2] L. A. Zadeh, "Fuzzy sets as a basis for a theory of possibility", Fuzzy Sets and Systems, 1 (1), pp. 3-28, 1978.

[3] R. Krishnapuram, J. M. Keller, "A possibilistic approach to clustering", IEEE Transactions on Fuzzy Systems, vol. 1 (2), pp. 98-110, 1993.

[4] J. C. Bezdek, Pattern Recognition with Fuzzy Objective Function Algorithms. New York: Plenum Press, 1981.

[5] F. Höppner, F. Klawonn, R. Kruse, T. Runkler, Fuzzy Cluster Analysis: Methods for Classification, Data Analysis and Image Recognition. Chichester: Wiley, 1999.

[6] J. C. Bezdek, J. M. Keller, R. Krishnapuram, N. R. Pal, Fuzzy Models and Algorithms for Pattern Recognition and Image Processing. New York: Springer, 2005.

[7] D. A. Viattchenin, A Heuristic Approach to Possibilistic Clustering: Algorithms and Applications. Heidelberg: Springer, 2013.

[8] K. T. Atanassov, "Intuitionistic fuzzy sets", Fuzzy Sets and
Systems, 20 (1), pp. 87-96, 1986.

[9] Z. Xu, Intuitionistic Fuzzy Aggregation and Clustering. Heidelberg: Springer, 2013.

[10] J. Kacprzyk, J. W. Owsinski, D. A. Viattchenin, S. Shyrai, “A new heuristic algorithm of possibilistic clustering based on intuitionistic fuzzy relations," Nowel Developments in Uncertainty Representation and Processing: Advances in Intuitionistic Fuzzy Sets and Generalized Nets - Proceedings of 14th International Conference on Intuitionistic Fuzzy Sets and Generalized Nets IWIFSGN'2015 (Cracow, Poland, October 26-28, 2015), K. T. Atanassov, O. Castillo, J. Kacprzyk, M. Krawczak, P. Melin, S. Sotirov, E. Sotirova, E. Szmidt, G. De Tré, eds., Heidelberg: Springer, 2015, 199-214.

[11] D. A. Viattchenin, S. Shyrai, "Intuitionistic heuristic prototype-based algorithm of possibilistic clustering", Communications on Applied Electronics, 1 (8), pp. 30-40, 2015.

[12] S. Shyrai, D. A. Viattchenin, "Clustering the intuitionistic fuzzy data. Detection of an unknown number of intuitionistic fuzzy clusters in the allotment", Proceedings of the International Conference on Information and Digital Technologies IDT'2015 (Zilina, Slovakia, July 7-9, 2015), Piscataway: IEEE Service Center, 2015, 302-311.

[13] W.-L. Hung, J.-S. Lee, C.-D. Fuh, "Fuzzy clustering based on intuitionistic fuzzy relations", International Journal of Uncertainty, Fuzziness and Knowledge-Based Systems, 12 (4), pp. 513-529, 2004.

[14] K. T. Atanassov, Intuitionistic Fuzzy Sets: Theory and Applications. Heidelberg: Springer, 1999.

[15] K. T. Atanassov, On Intuitionistic Fuzzy Sets Theory. Heidelberg: Springer, 2012.

[16] E. Szmidt, Distances and Similarities in Intuitionistic Fuzzy Sets, Heidelberg: Springer, 2014.

[17] A. Kaufmann, Introduction to the Theory of Fuzzy Sets, New York: Academic Press, 1975.

[18] Z. Wang, Z. Xu, S. Liu, J. Tang, “A netting clustering analysis method under intuitionistic fuzzy environment", Applied Soft Computing, 11 (8), pp. 5558-5564, 2011.

[19] D. A. Viattchenin, "A method of construction of intuitionistic fuzzy tolerances based on a similarity measure between intuitionistic fuzzy sets", New Developments in Fuzzy Sets, Intuitionistic Fuzzy Sets, Generalized Nets and Related Topics - Proceedings of 10th International Workshop on Intuitionistic Fuzzy Sets and Generalized Nets IWIFSGN'2011 (Warsaw, Poland, September 30, 2011), Vol. I: Foundations, K. T. Atanassov, M. Baczyński, J. Drewniak, J. Kacprzyk, M. Krawczak, E. Szmidt, M. Wygralak, S. Zadrożny eds., Warsaw: IBS PAN, 2012, pp. 191-202.

[20] J. Kacprzyk, D. A. Viattchenin, S. Shyrai, E. Szmidt, “A nowel similarity measure between intuitionistic fuzzy sets for constructing intuitionistic fuzzy tolerance," Nowel Developments in Uncertainty Representation and Processing: Advances in Intuitionistic Fuzzy Sets and Generalized Nets Proceedings of 14th International Conference on Intuitionistic Fuzzy Sets and Generalized Nets IWIFSGN'2015 (Cracow, Poland, October 26-28, 2015), K. T. Atanassov, O. Castillo, J. Kacprzyk, M. Krawczak, P. Melin, S. Sotirov, E. Sotirova, E. Szmidt, G. De Tré, eds., Heidelberg: Springer, 2015, 175-183. 